Published in final edited form as:

Angew Chem Int Ed Engl. 2019 March 04; 58(10): 3067-3072. doi:10.1002/anie.201811691.

\title{
Molecular scaffolds as double targeting agents for the diagnosis and treatment of Neuroblastoma
}

\author{
Gonzalo Villaverde $^{[a]}$, Arantzazu Alfranca ${ }^{[b],[c]}$, Dr. África Gonzalez-Murillo[c],[d], Dr. Gustavo \\ J. Melen ${ }^{[\mathrm{c}],[\mathrm{d}]}$, Rafael R. Castillo[a], Dr. Manuel Ramírez ${ }^{[\mathrm{c}],[\mathrm{d}]}$, Dr. Alejandro Baeza ${ }^{[\mathrm{a}],[\mathrm{e}],{ }^{*}, \text { Prof. }}$ \\ María Vallet-Regi[a], ${ }^{*}$ \\ [a]Dpto. Química en Ciencias Farmacéuticas. Facultad de Farmacia, Universidad Complutense de \\ Madrid. Plaza Ramon y Cajal s/n. Instituto de Investigación Sanitaria Hospital 12 de Octubre i+12 \\ ¡ Centro de Investigación Biomédica en Red de Bioingeniería, Biomateriales y Nanomedicina \\ (CIBER-BBN) Madrid, Spain \\ ${ }^{[b]}$ Servicio de Inmunología. Hospital Universitario de La Princesa \\ [c] Instituto de Investigacion Sanitaria La Princesa. Diego de León, 62. 28006 Madrid, Spain \\ [d]Servicio de Hematología y Oncología Pediátrica, Hospital Infantil Universitario Niño Jesús, \\ Madrid, Spain \\ [e]Dpto. Materiales y Producción Aeroespacial, ETSI Aeronáutica y del Espacio, Universidad \\ Politécnica de Madrid, 28040-Madrid, Spain
}

\begin{abstract}
The selective delivery of therapeutic and imaging agents to tumoral cells has been postulated as some of the most important challenges in nanomedicine field. The cellular selectivity of nanocarriers is usually achieved by their surface decoration with targeting moieties able to recognize these cells in a specific manner. Neuroblastoma (NB) is the most frequent extra-cranial pedriatic tumor, almost incurable at advanced stages. Meta-iodo-benzilguanidine (MIBG) is widely used for the diagnosis of this disease due to its strong affinity by the norepinephrine transporter (NET) usually overexpressed on the membrane of the malignant cells. Herein, a complete family of novel Y-shaped scaffolds which contains structural MIBG analogues covalently attached in each end of the Y-structure has been synthesized. The enhanced cellular uptake capacities of these double-targeting ligands have been evaluated in vitro and in vivo yielding one specific Y-shape structure which presents capacity to be engulfed by the malignant cells and be accumulated in the tumoral tissue, significantly higher than the structure which contains only one single targeting agent. This Y-shaped ligand can provide a powerful tool for the current and treatment diagnosis of this disease.
\end{abstract}

\footnotetext{
*vallet@ucm.es abaezaga@ucm.es.
}

This manuscript has been accepted after peer review and appears as an Accepted Article online prior to editing, proofing, and formal publication of the final Version of Record (VoR). This work is currently citable by using the Digital Object Identifier (DOI) given below. The VoR will be published online in Early View as soon as possible and may be different to this Accepted Article as a result of editing. Readers should obtain the VoR from the journal website shown below when it is published to ensure accuracy of information. The authors are responsible for the content of this Accepted Article. 


\section{Keywords}

neuroblastoma; nanomedicine; targeting; meta-iodobenzylguanidine; molecular scaffold

Neuroblastoma (NB) is the most common extra-cranial solid tumor in childhood. The 50\% of the cases are diagnosed at late stages when the disease has spread throughout the entire body, in metastatic stage. ${ }^{[1]}$ The treatment of metastatic NB (stage IV) is usually based on the combination of various chemotherapeutic agents in high doses, together with surgical removal of the primary tumor, only in cases of a good response to the initial chemotherapy. Finally, the treatment is completed with radiotherapy, or complementation of the initial chemotherapy with autologous for hematopoietic rescue and pre-treatment of possible residual disease with differentiating agents. ${ }^{[2]}$ Despite this multimodal treatment, up to $60 \%$ of children with stage IV of NB relapse. Thus, the prognosis of advanced NB is very poor, which demonstrates the inability of the current treatment to eradicate the entire disease. NB cells come from the neural crest, such as precursors of the sympathetic nervous system. Around $95 \%$ of NB cells present an overexpression of the transmembrane protein norepinephrine transporter (NET) being able of capturing norepinephrine precursors. The synthetic norepinephrine analogue meta-iodobenzylguanidine (MIBG) selectively binds to NET receptor and for this reason, it is widely used for the diagnosis of NB when the molecule is labeled with a radioactive iodine. ${ }^{[3],[4][5]}$ Several structure/activity relationship (SAR) studies have demonstrated that benzene ring and guanidine moiety are the pillars for maintaining the NET binding capacity of MIBG. Thus, the rest of the structure admits some degree of modification such as substitution of the iodine for other group with different electro-donor / attractor character. Further, changes in the position of the substitution and even replacing the aromatic ring itself have been carried out for improving both, cell uptake and tumor tissue accumulation. ${ }^{[6-10]}$ However, the in vivo assays did not yield the expected results, being the MIBG the most active. Even, the incorporation of a second guanidine group in position 4 led to a significant decrease in the internalization in NB cells. ${ }^{[11]}$ Until now, only the incorporation of a methyl group in position 4 improves the retention of this molecule by the NB cells. ${ }^{[7-9,11]}$ The MIBG analogue which contains an amino group instead of the iodine, meta-aminobenzylguanidine (MABG), has shown similar NET binding ability that MIBG. For this reason, MABG was conjugated to non-radioactive fluorescence compounds in the near infrared by organic spacers acting as image agent, ${ }^{[12]}$ resulting an excellent vectorization agent that yields improved spatial imaging resolution in comparison to that obtained by SPECT. Furthermore, we previously employed MABG as targeting moiety for guiding nanoparticles specifically to neuroblastoma in murine models. ${ }^{[13]}$ In this work, MABG was anchored on the surface of mesoporous silica nanoparticles (MSN) through a polyethylene glycol polymer (PEG) of $2000 \mathrm{Da}$ of molecular weight which acted as spacer between the nanoparticle surface and the recognition moiety. The presence of this targeting group enhanced the nanoparticle uptake in NB cell around 4-fold. Additionally, it was found that the presence of this targeting group on the nanoparticle surface, led to a selective accumulation and high retention of the nanocarriers in the tumoral mass of a xenograft mice model whereas untargeted to the rapid excretion of the nanoparticles. This approach is based on the use of one single targeting agent which interacts with a membrane cell receptor overexpressed in the tumoral cells. Nature usually employs antibodies as 
recognition agents and these proteins present a Y-shaped structure able to bind to the antigens by two points instead of one. ${ }^{[14]}$ Inspired by these natural molecules, the following two questions were raised to the authors; synthetic Y-shaped scaffold which contains two MABG-based binding points could improve even more the cellular uptake within NB cells?, and the second question related to this first one, which is the optimal Y-structure that yielded the best NET-binding capacities? The present work describes the synthesis and biological evaluation of different Y-shaped scaffolds decorated with MABG analogues, and also with other usually employed targeting moieties, for the look out of improved vectorization agents which allow the therapeutic / imaging targeted delivery to neuroblastoma. ${ }^{[15]}$ This Y shape promising prototype could pave the way of a safer delivery of chemotherapy and also for a more sensitive and efficient diagnosis of NB lesions. Previous to the construction of Y-shape moieties, we decided to synthesize a small collection of MABG-based derivatives in order to select the analogue which yielded the higher cellular uptake in NB. These experiments will be essential in order to choose the best candidate for its introduction in the Y shape scaffold. Thus, different MABG analogues which carry halogens attached to the benzyl ring or with the amide group in meta- or para- position were synthesized. Then, these molecules were conjugated with a fluorescein-labeled polyethylene glycol (PEG) chain of $3500 \mathrm{Da}$ of molecular weight (Figure 1) The PEGylated fluorescein acts as fluorescent marker payload emulation which makes possible their tracing on in vitro assays by flow cytometry (fluorescein ( $\chi \mathrm{ex}=492 \mathrm{~nm}$; $\chi \mathrm{em}=520 \mathrm{~nm}$ ). Thus, the marked single derivates, F-PEG-S3, ( $m$-glycinamido-benzylguanidine), F-PEG-S4 ( $p$-glycinamido-benzylguanidine), F-PEG-SCl ( $p$-chloro- $m$-glycinamido-benzylguanidine) and F-PEG-SI, ( $p$-glycinamido- $m$-iodobenzylguanidine)) were synthetized from the corresponding compounds with terminal primary alkyl amine group proportioned by glycine coupling. (See Supporting information (SI) from Scheme S1 to Scheme S5). The cellular uptake of these conjugates was evaluated in the NB cell line (NB1691-luc). Cells were incubated for 2 hours with each of these conjugates at $50 \mu \mathrm{g} \cdot \mathrm{mL}^{-1}$ in culture medium, washed once with PBS and the well refilled with fresh medium. Finally, cells were incubated overnight and washed with PBS for removing the non-incorporated conjugate. The cellular uptake was measured by flow cytometry showing that the introduction of halogens in the benzyl ring yielded lower internalization capacity whereas the system which presents only one amide group (both in meta- and para- position) achieved the best results. After 2 hours of incubation with the corresponding analogue the para-amide substituted compound (S4) showed the best performance with $75 \%$ of cells with fluorescence inside, versus the barely $50 \%$ obtained by the meta- analogue(S3). Thus, para- and meta-amino derivatives were selected as the best systems to be incorporated in the Y-shaped structures. As a first approximation for doubling the uptake properties of the MAGB analogues, flexible medium length amino acid Y-shape scaffold (DF) was chosen as initial candidate to combine the benzylguanidine analogues to each other. On the other hand, other motives, were also tested in combination with the MABG analogues in the DF scaffold. DF structure is based on a two Lysine units connected by a peptidic bond. Both targeting agents were connected to the scaffold by amidation of the primary amine groups. For this aim succinic derivative of S3 and S4 were synthetized previously (SI, Scheme S6) in order to afford DF3 and DF4. Besides these systems, DFS3TPP and DFS3-RGD were also synthetized. DFS3-TPP was designed with the two unit peptide scaffold connecting the succinic $\mathrm{S} 3$ analogue and triphenilphosphine unit, that 
present vectorization to the mitochondria. ${ }^{[16],[17],[18]}$ On the other hand, DFS3-RGD, was constructed with the same structure changing the motif for the tripeptide RGD trying to combine two different tumoral receptors that will be present in the NB tumor endothelium and cell membrane. ${ }^{[19]}$ (See SI Scheme S12 and S13). In all cases the system have a primary amine group as exclusive anchoring point to link with the pre-activated acid group from PEG, which connected to the corresponding payload. Similar to the mentioned analogues, a fluorescent moiety was anchored for following the system in in vitro experiments (F-PEGDFX). Figure S7 from SI shows how double systems DF3 and DF4 present more than $90 \%$ of cellular uptake at $50 \mu \mathrm{g} . \mathrm{mL}^{-1}$ and even at $25 \mu \mathrm{g} \cdot \mathrm{mL}^{-1}$. Following the tendency, the single S4 showed an excellent results with a $75 \%$, leading the rest of single ligands which presented an average of $50 \%$ of internalization at the higher concentration. The behavior of the double systems DF3-TPP and DF3-RGD, with a notable decrease of internalization versus the double DF3 and DF4, even worse than the single agents S3 and S4, showed that the introduction of nonspecific moieties in the system could interfere with the binding properties of the benzylguanidine group and into the recognition process with the NET. These data made the authors to stake out the final aim of the work and focus their efforts on obtaining the best benzylguanidine based $\mathrm{Y}$ shape multivalent ligand applicable as vectorization agent for NB. For this aim, studies about the influence of the length and flexibility of the scaffold on their uptake properties were carried out for the following FPEGylated double ligands families classified according with their rigid or flexible nature and their length (Figure 1 and Figure 2 a): Double rigid (DR3, DR4), Double flexible small (DFS3, DFS4), Double flexible (DF3, DF4), and double flexible large (DFL3, and DFL4).

Double Rigid scaffolds (DR) are based on aromatic core body. They were synthetized by amide formation coupling reaction with same starting material described for single analogues connecting two units of S3 and S4 with terminal alkyl amine group with isophthalic acid analogue for affording the homogeneous DR3 and DR4, respectively. (See SI Scheme S7 and S8). Double Flexible ligands (DF) are based on the presence of an oligopeptidic chain. Among this group, three different types of flexible structures were synthesized according with their lengths, presenting one, two and up to three amino acids, DFS, DF, DFL, respectively. Briefly, these DF analogues were synthesized by coupling amidebezylguanidine analogues in meta- and para- conformation (S3 and S4) through a peptide chain, affording DFS3 and DFS4, with one amino acid in the structure, DF3 and DF4 with two amino acids, and DFL3 and DFL4, with three amino acids conferring more flexibility and distance between the binding points with the increase of units in the scaffold structure. DF ligands were synthetized through typical solid phase peptide synthesis, by conventional Fmoc deprotection/HOBT-based acid activation coupling starting from FmocNH-Lys-NH-Mtt Wang resin. The rest of amino acids, as well as the targeting agents previously synthetized in this case with terminal carboxylic acid group proportioned by succinic acid coupling with the corresponding initial analogue S3 and S4 (See SI from Scheme S9 to S11 and Scheme S6), were consecutively coupled. Finally, the complete systems were released from the resin by the addition of TFA cocktail. In vitro experiments for internalization in NB cells, were carried out by screening the PEGylated and fluorescein marked analogues for each family at $12.5 \mu \mathrm{g} / \mathrm{ml}$ and $6.5 \mu \mathrm{g} / \mathrm{ml}$ following conditions previously described. The analogues analyzed were: S3 and S4, as references, plus, rigid 
ligands DR3 and DR4, and finally from flexible scaffolds, DFS3, DFS4, DF3, DF4 and both DFL3 and DFL4.

As Figure 2. b shows, the act of doubling the number of targeting moieties did not govern the entire uptake process. Moreover instead of number of targeting agent, their disposition, their distance to each other and their freedom to adopt cluster configuration in their interaction with the receptor in the cell membrane seemed determinant. Thus, the effect of the structure and length of the scaffold has a dominant effect in order to interact with the NET of the NB cell membrane. Taking as an example the data for the meta-derivate, double rigid scaffold DR3 improved the internalization properties in front of its corresponding single counterpart S3 barely at same scale to flexible analogues DF3. This equal behavior may be explained by the duplication of the interaction points with NET in both analogues, further they present equal distance between recognition points following MM2 calculations (DR3 (7 $\AA$ ), DF7 (8 $\mathbf{\AA})$ ). Thus, in this case, the effect in the uptake process is governed by the duplication of targeting agent. On the other hand, the larger and more flexible analogue, DFL3 (16 ̊, of distance between recognition points)), resulted significantly the more efficient in the uptake process compared to S3 and the rest of the ligands in the family. Thus, the fact of having two benzyl guanidine derivate in the same system sharpens when the distance between the recognition moieties is the barely three amino acids and the system is flexible.

Para analogues from Figure 2a kept a similar behavior, when the distance is about 16.9 Angstrom (DFL4) the internalization folds up around 3 times versus their double more rigid (DR4) and flexible shorter families (DF and DFS) counterparts. This fact gave light for understanding the behavior of the targeting ligand. The distance and the flexibility of the ligand allow the system to work such as, common bio- receptor with double anchoring point, and if the distant and flexibility of the ligand is not appropriate the double ligand works such as single targeting agent interacting with only one of the recognitions points present in the scaffold. Thus, comparing the uptake properties of the best bi-functional system (DFL4) with the best mono-functional (S4) we can conclude that for $100 \%$ of internalization in NB cell, DFL4 analogue needs 10 times less concentration than S4. Once known the behavior in terms of cell uptake, the next step to corroborate the characteristics of these systems, was to study the selective transporting and accumulation of significate payload (drugs, nanoparticles or even image agents) to the neuroblastoma tumoral environment. To achieve this goal, we conducted in vivo experiments to compare ligands with the best performance in vitro (i.e. DFL3 and DFL4) versus a non-targeted system. To this end, mesoporous silica nanoparticles (MSN's) marked with Cy7 were synthetized and surface-functionalized with the corresponding vectorization agents, through a PEG linker chain affording the control naked material NP-NH${ }_{2}$ and the targeted nanoparticles NP-PEG-DFL3 and NP-PEG-DFL4.

Previous results of our group have shown that MSN's are highly efficient nanotransporters for drug delivery, due to their optimal loading and controlled release properties.

Additionally, MSN's may be easily functionalized for adding vectorization agents. ${ }^{[20-22]}$. $\mathrm{Cy} 7$ is a near infrared marker frequently used for in vivo assays, given the reduced tissue auto fluorescence at this wavelength. This characteristic makes $\mathrm{Cy} 7$ a suitable candidate to label the nano-transporters for their in vivo evaluation. We carried out functionalization of 
the agents to the nanoparticle's surface through a PEG linker; PEG confers more ability in dispersion and helps to abolish the opsonization process which may prolong circulation time in the blood stream. ${ }^{[23]}$ We subcutaneously implanted human neuroblastoma NB1691-luc cells $\left(2 \times 10^{6}\right.$ cells/mouse) into the flank of immuno-deficient NGS mice (NOD/SCID deficient in the common gamma chain). These cells constitutively express Luciferase, which allows in vivo monitoring of tumor growth by bioluminescence quantification upon injection of Luciferase substrate Luciferin (1,25 mg/mouse). Thus, three weeks after cell inoculation we assessed tumor development in mice, and sorted mice into three groups with a homogeneous distribution of tumor size among them (3-5 specimens per group (see Figures $\mathrm{S} 9$ and $\mathrm{S} 10$ in SI). Mice received intravenous injections of $1 \mathrm{mg}$ of $\mathrm{NP}^{-\mathrm{NH}_{2}}$, NP-PEGDFL3, or NP-PEG-DFL4 in $0.2 \mathrm{~mL}$ of saline solution. In order to evaluate system accumulation in the tumor mass in each case, we quantified fluorescent signal ratio of $\mathrm{Cy} 7$ in tumor area vs. the whole mouse, at 72 hours after system administration. As shown in Figure 3, we observed preferential accumulation of targeted MSN's systems (NP-PEGDFL3 and NP-PEG-DFL4) in tumor mass, which was detected by bioluminiscence. In conclusion, this work has allowed the selection of a novel system based on a Y-shape multivalent double ligand targeting for NB with a specific composition and length. Their behavior in terms of selectivity and efficacy for improving cell internalization and in vivo accumulation in neuroblastoma xenografts have been evaluated. These new Y-shape double system presents a substantial improvement in cell uptake in vitro, 20-times higher than the single-targeted system and an effective accumulation in tumor mass in vivo. Further, they are easily tunable for using as versatile targeting system for drug delivery in conjugates or nanotransporters, as well as for imaging agents. Our findings may pave the way for rising the efficacy against NB in terms of diagnosis or treatment.

\section{Supplementary Material}

Refer to Web version on PubMed Central for supplementary material.

\section{Acknowledgements}

MVR thank the funding from the European Research Council through the Advanced Grant VERDI (ERC-2015 AdG proposal no. 694160) and Asociación NEN. Financial support from Ministerio de Economía y Competitividad, (MEC), Spain (Project MAT2015-64831-R) is gratefully acknowledged. AA is supported by grant FIS PI15/01491. MR is supported by Asociación NEN, Asociación Pablo Ugarte and Fundación Neuroblastoma.

\section{References}

[1]. Maris JM. N Engl J Med. 2010; 362:2202-2211. [PubMed: 20558371]

[2]. Pinto NR, Applebaum MA, Volchenboum SL, Matthay KK, London WB, Ambros PF, Nakagawara A, Berthold F, Schleiermacher G, Park JR, et al. 2015; 33doi: 10.1200/JCO. 2014.59.4648

[3]. Tumors N, Carlin S, Mairs RJ, Mccluskey AG, Tweddle DA, Sprigg A, Estlin C, Board J, George RE, Ellershaw C, et al. 2003; 9:3338-3344.

[4]. Matthay KK, George RE, Yu AL. Clin Cancer Res. 2012; 18:2740-53. [PubMed: 22589483]

[5]. Schlessinger A, Geier E, Fan H, Irwin JJ, Shoichet BK, Giacomini KM, Sali A. Proc Natl Acad Sci U S A. 2011; 108:15810-5. [PubMed: 21885739]

[6]. Hadrich D, Berthold F, Steckhan E, Bönisch H. J Med Chem. 1999; 42:3101-8. [PubMed: 10447954] 
[7]. Vaidyanathan G, Shankar S, Affleck DJ, Welsh PC, Slade SK, Zalutsky MR. 2001:798-806.

[8]. Vaidyanathan G, Shankar S, Zalutsky MR. 2001:786-797.

[9]. Vaidyanathan G, Welsh PC, Vitorello KC, Snyder S, Friedman HS, Zalutsky MR. Eur J Nucl Med Mol Imaging. 2004; 31:1362-70. [PubMed: 15205923]

[10]. Streby KA, Shah N, Ranalli MA, Kunkler A, Cripe TP. Pediatr Blood Cancer. 2015; 62:5-11. [PubMed: 25175627]

[11]. Vaidyanathan G, Shankar S, Affleck DJ, Alston K, Norman J, Welsh P, LeGrand H, Zalutsky MR. Bioorg Med Chem. 2004; 12:1649-56. [PubMed: 15028258]

[12]. Wang W, Shohet J, Mawad M, Ke S. Non-Radioactive Agents for Neuroblastoma Imaging. 2011

[13]. Villaverde G, Baeza A, Melen GJ, Alfranca A, Ramirez M, Vallet-Regí M. J Mater Chem B. $2015 ; 3: 4831-4842$.

[14]. Quynh T, Nguyen N, Lim KW, Phan AT. 2017; 33:1-7.

[15]. Yao VJ, Angelo SD, Butler KS, Theron C, Smith TL, Marchiò S, Gelovani JG, Sidman RL, Dobroff AS, Brinker CJ, et al. J Control Release. 2016; 240:267-286. [PubMed: 26772878]

[16]. Luo G-F, Chen W-H, Liu Y, Lei Q, Zhuo R-X, Zhang X-Z. Sci Rep. 2014; 4:1-10.

[17]. Kang BH, Plescia J, Song HY, Meli M, Colombo G, Beebe K, Scroggins B, Neckers L, Altieri DC. J Clin Invest. 2009; 119(3):454-464. [PubMed: 19229106]

[18]. Chen W-H, Xu X-D, Luo G-F, Jia H-Z, Lei Q, Cheng S-X, Zhuo R-X, Zhang X-Z. Sci Rep. 2013; 3:3468. [PubMed: 24336626]

[19]. Rosca EV, Stukel JM, Gillies RJ, Vagner J, Caplan MR. 2007:3830-3835.

[20]. Vallet-Regi M, Rámila A, del Real RP, Pérez-Pariente J. Chem Mater. 2001; 13:308-311.

[21]. Vallet-Regí M, Ruiz-Hernández E. Adv Mater. 2011; 23:5177-218. [PubMed: 22009627]

[22]. Baeza A, Colilla M, Vallet-Regí M. Expert Opin Drug Deliv. 2014:1-19.

[23]. Meng H, Liong M, Xia T, Li Z, Ji Z, Zink JI, Nel AE. ACS Nano. 2010; 4:4539-50. [PubMed: 20731437] 

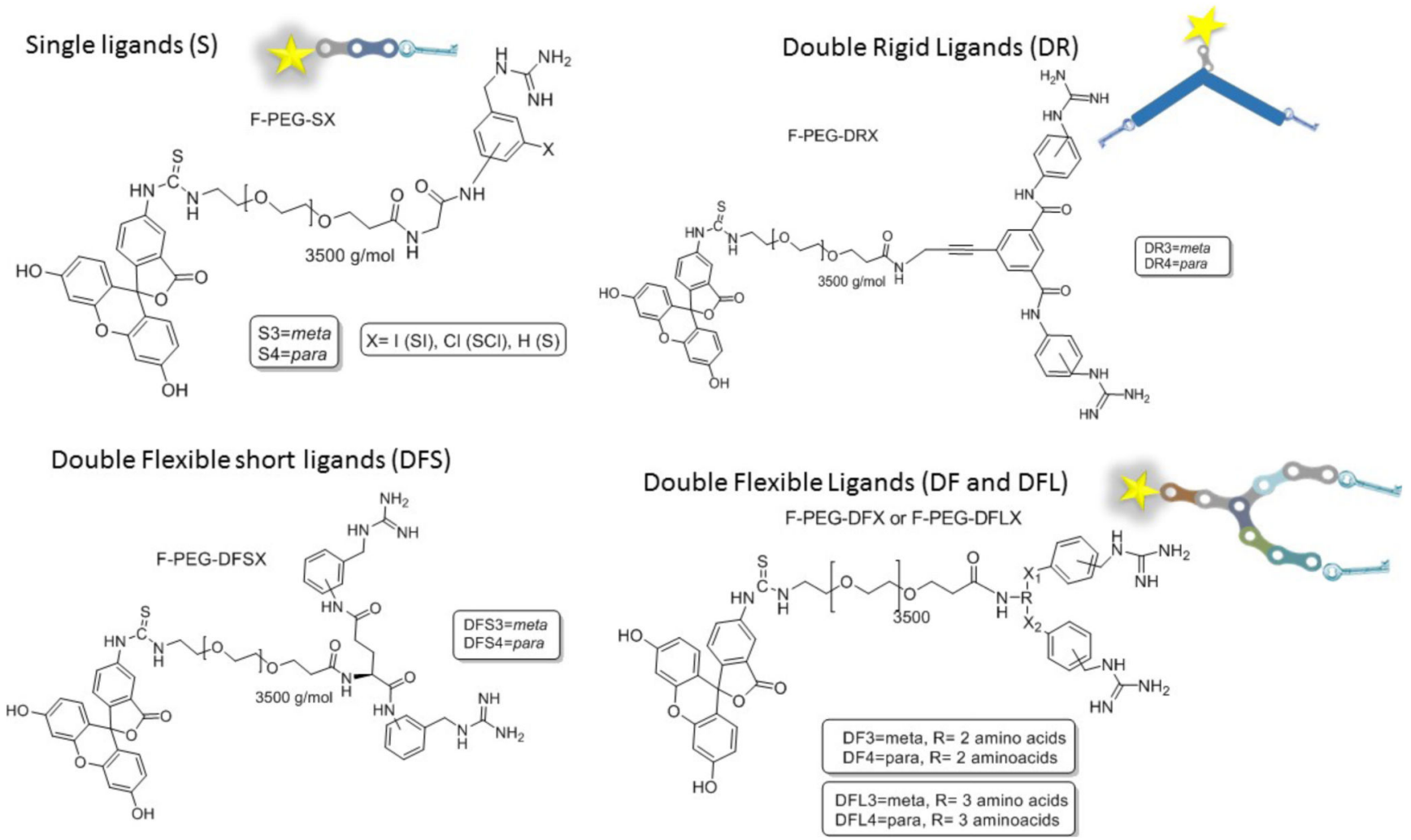

Figure 1.

Fluorescein PEGylated analogues for in vitro analysis from all families of single and double targeting system. $\mathrm{X}_{1}$ and $\mathrm{X}_{2}=$ amide bond in all cases. 

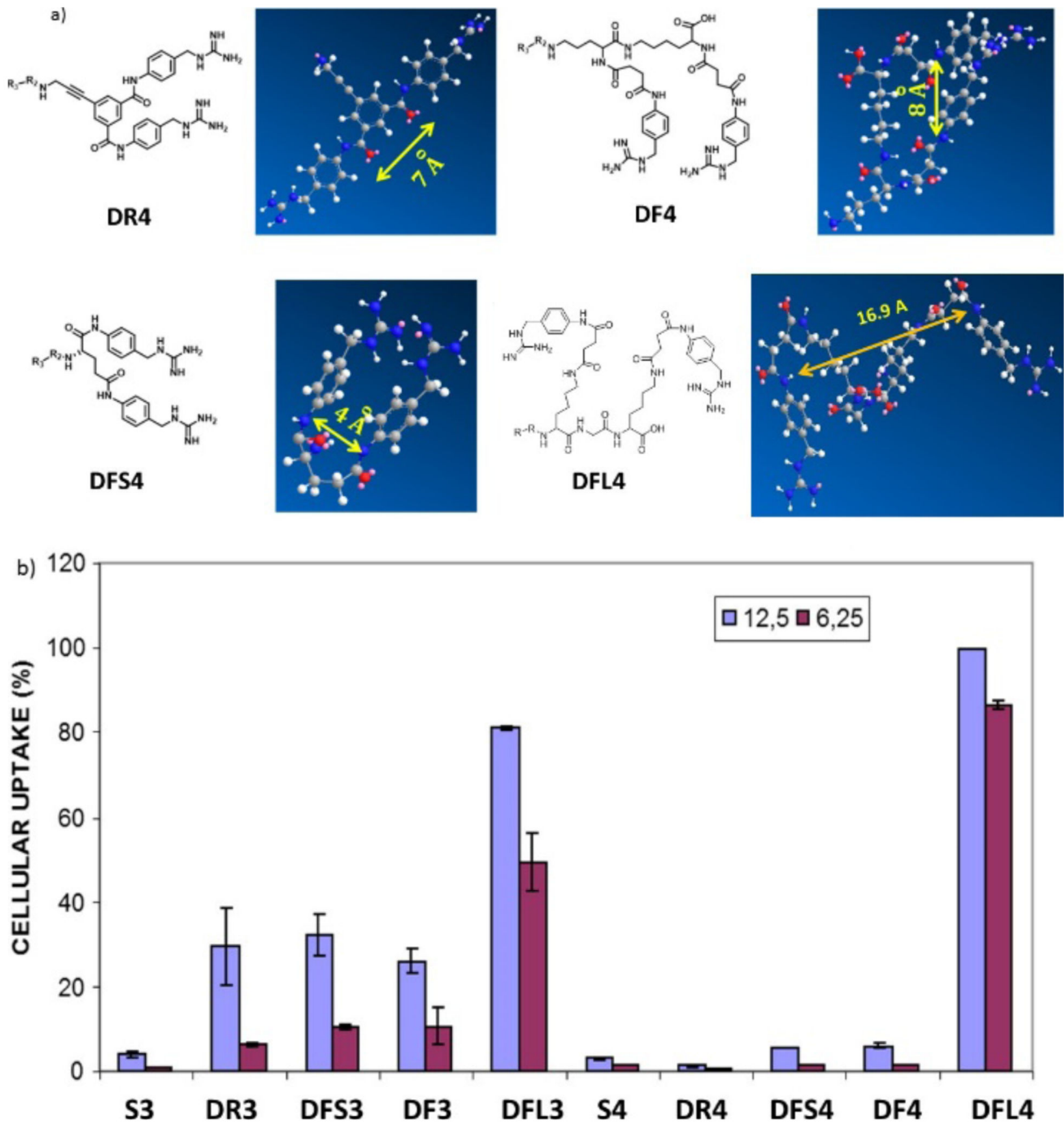

Figure 2.

a) Calculated distance by molecular mechanics (MM2) calculations between recognition spots in the double scaffolds structures DR4 (7 ̊), DFS4 (4 ̊), DF4 (8 ̊) and DFL4 (16.9 $\AA)$, NOTE: The distances are equivalent in the case of meta-substituted systems b) Initial screening of single (S3 and S4) versus double ligands for uptake in NB cells at low concentrations, $12.5 \mu \mathrm{g} . \mathrm{mL}-1$ (blue) and $6.25 \mu \mathrm{g} . \mathrm{mL}-1$ (red). 
tumor/whole $72 \mathrm{~h}$

a)

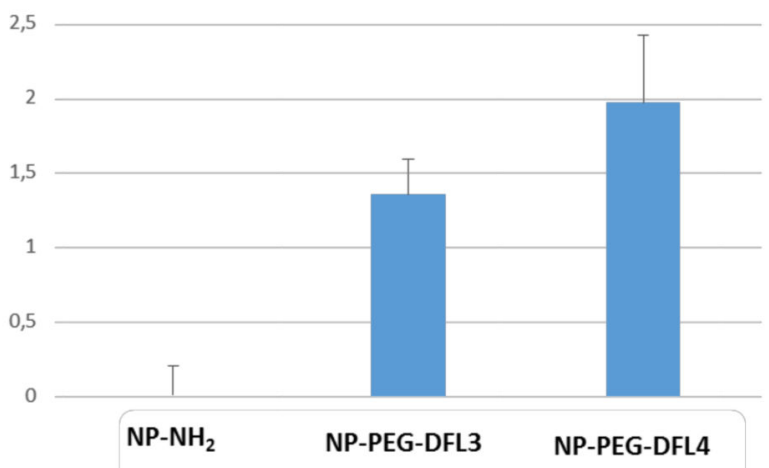

\section{I. $\mathrm{NP}-\mathrm{NH}_{2}$}

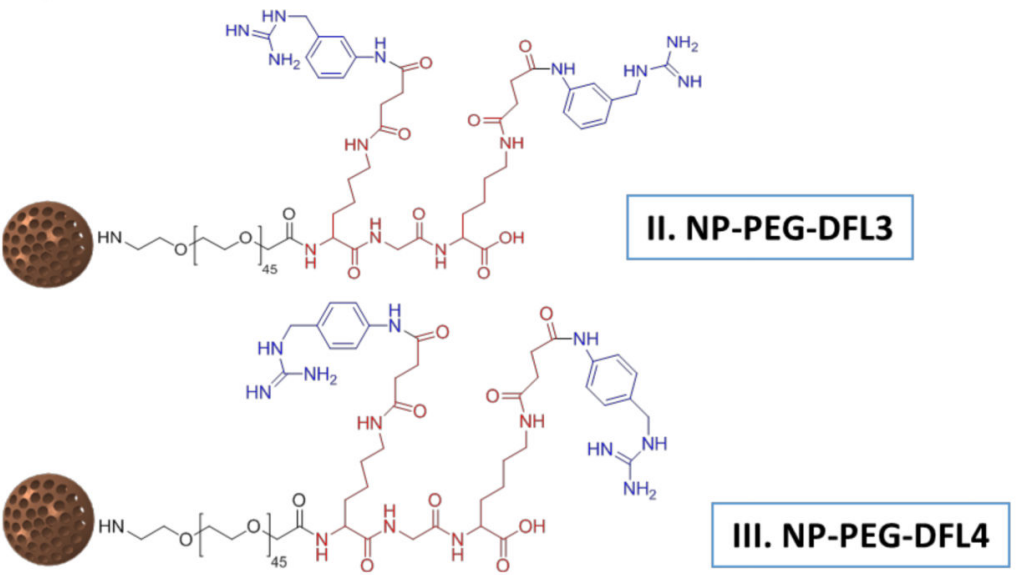

b)
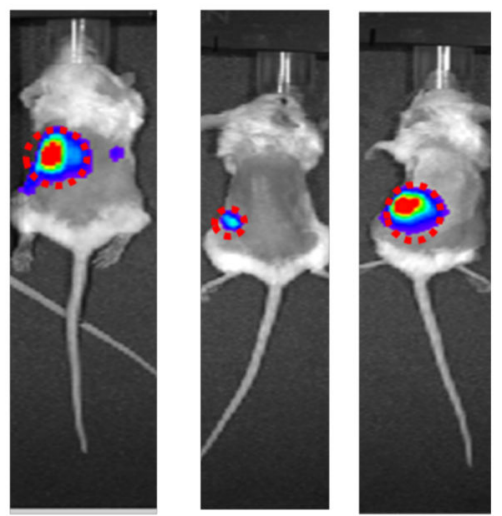

High Intensity
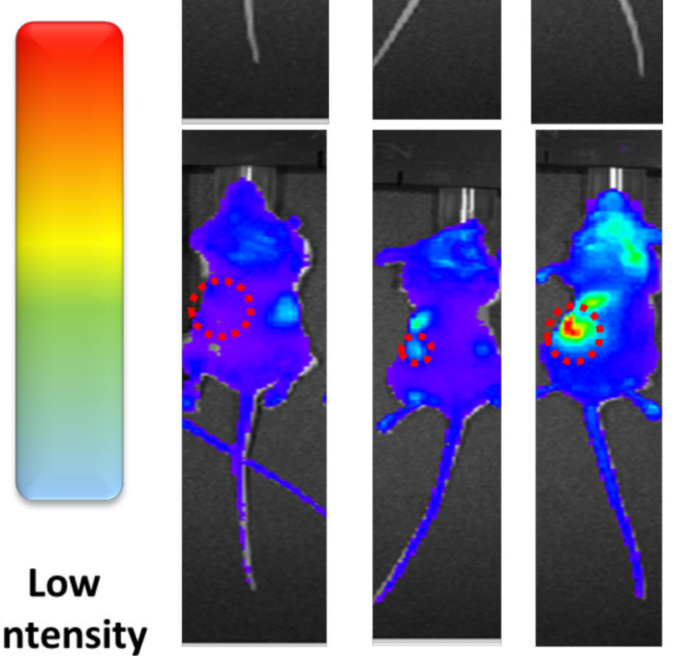

I

Figure 3.

a) Upper panel, Graphic shows relative fluorescent signal (tumor area/whole mouse) obtained from xenograft neuroblastoma-inoculated mice $72 \mathrm{~h}$ after injection with either NPNH2, NP-PEG-DFL3, or NP-PEG-DFL4 (mean \pm SD; $n=3-5$ per group); lower panel b) Representative photographs of xenograft mice treated as in a), showing luminescent tumor signal (upper panels) and fluorescent nanoparticle signal (lower panels) 